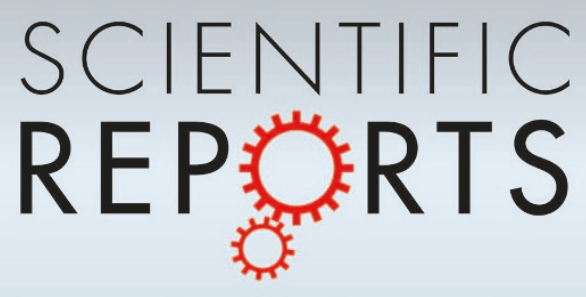

\title{
OPEN \\ Giant electrocaloric effect in ferroelectric nanotubes near room temperature
}

SUBJECT AREAS:

NANOSCALE MATERIALS

COMPUTATIONAL SCIENCE

Received

25 September 2014

Accepted

9 December 2014

Published

12 January 2015

Correspondence and requests for materials should be addressed to J.W. (jw@zju.edu.cn)

\author{
Man Liu \& Jie Wang
}

Department of Engineering Mechanics, School of Aeronautics and Astronautics, Zhejiang University, Hangzhou 310027, China.

Ferroelectric perovskite oxides possess large electrocaloric effect, but only at high temperature, which limits their potential as next generation solid state cooling devices. Here, we demonstrate from phase field simulations that a giant adiabatic temperature change exhibits near room temperature in the strained ferroelectric $\mathrm{PbTiO}_{3}$ nanotubes, which is several times in magnitude larger than that of $\mathrm{PbTiO}_{3}$ thin films. Such giant adiabatic temperature change is attributed to the extrinsic contribution of unusual domain transition, which involves a dedicated interplay among the electric field, strain, temperature and polarization. Careful selection of external strain allows one to harness the extrinsic contribution to obtain large adiabatic temperature change in ferroelectric nanotubes near room temperature. Our finding provides a novel insight into the electrocaloric response of ferroelectric nanostructures and leads to a new strategy to tailor and improve the electrocaloric properties of ferroelectric materials through domain engineering.

T

he electrocaloric effect (ECE) can be described as the temperature change of a material under adiabatic condition in response to an applied electric field which is the converse pyroelectric effect that exists in all ferroelectric materials ${ }^{1}$. Due to their significantly temperature-dependent spontaneous polarization, ferroelectric materials based on the perovskite-structured oxides possess large ECE, and have been considered as prime candidates for solid state cooling devices ${ }^{2-4}$. In particular, the recent finding of giant ECE near the ferroelectric transition temperature in the $\mathrm{Pb}(\mathrm{Zr}, \mathrm{Ti}) \mathrm{O}_{3}$ thin films ${ }^{5}$ makes this field has been drawn considerable attention.

On the other hand, with the miniaturization of modern electronic devices, cooling these devices with high performance becomes a challenge issue due to the small size. One of the possible solutions would be the application of the ECE of ferroelectric nanostructures or low-dimensional ferroelectrics, such as ferroelectric ultrathin films, nanotubes and nanodots. For low-dimensional ferroelectrics, the enhanced depolarization field and strong polarization-strain coupling can alter the polarization distribution, the ferroelectric transition temperature, the dielectric, pyroelectric as well as electrocaloric properties ${ }^{6-8}$, which makes them completely different from their bulk counterparts ${ }^{9-10}$. To understand the ECE of low-dimensional ferroelectrics, there are extensive studies on ferroelectric thin films in which the ECE can be tuned through epitaxial strain, composition, temperature and electric field ${ }^{11-14}$.

Although the ECE of ferroelectric thin films has been extensively investigated, the ECE for other low dimensional ferroelectrics, such as ferroelectric nanotubes and nanodots, has seldom been investigated in the literature. The cooling devices with low dimensional ferroelectrics and high performance are essential for the development of next generation nano-electronic chips. Furthermore, low dimensional ferroelectrics, such ferroelectric nanotubes, have been successfully fabricated in experiments ${ }^{15-17}$, which makes it possible to be used in the nano ECE cooling devices. Differing from the planar ferroelectric thin film, the unique geometry of ferroelectric nanotubes provides more dedicated interplay among the depolarization field, strain, temperature and polarization, which may induce novel property and high performance. Therefore, it is of fundamental significance and technologic importance to understand the ECE of ferroelectric nanotubes.

In general, the ECE of ferroelectrics intrinsically comes from the temperature dependence of polarization ${ }^{18}$, which becomes significant only near the ferroelectric phase transition where the polarization undergoes a dramatic change with temperature. The ferroelectric phase transition temperature is often much higher than room temperature, which limits the application of ferroelectrics in solid state cooling devices. In addition to the intrinsic contribution, the ECE of ferroelectrics is also dependent on the extrinsic contribution due to the change of domain structures ${ }^{19-20}$. Our previous work ${ }^{21}$ shows that the extrinsic contribution from the domain transition driven by temperature can significantly enhance the $\mathrm{ECE}$ of bulk $\mathrm{PbTiO}_{3}$ ferroelectrics subjected to an external strain. Nevertheless, the large ECE induced by domain transition exhibits only in high-temperature range although it is below the Curie temperature of bulk $\mathrm{PbTiO}_{3}$ ferroelectrics. The domain transition temperature is usually close to the ferroelectric transition temperature. Due to the enhancement of depolarization field, the 


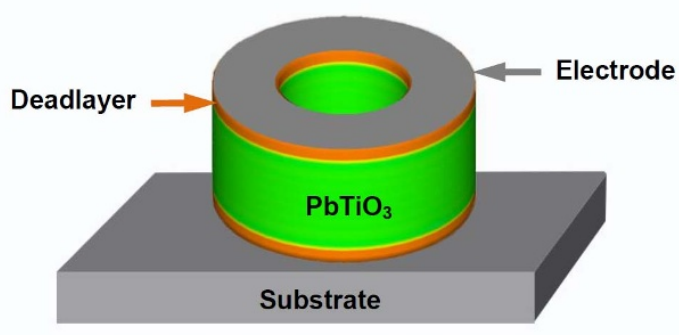

Figure 1 Schematic illustration of a $\mathrm{PbTiO}_{3}$ ferroelectric nanotube with upper and lower electrodes epitaxially grown on a substrate. Two deadlayers at the upper and lower interfaces are included to model the material degradation at electrode-ferroelectrics interface.

ferroelectric transition temperature decreases when the size of ferroelectrics decreases ${ }^{22}$. Correspondingly, the domain transition temperature may also decrease with the decrease of size, implying that large ECE is possible to be induced by domain transition near room temperature. In the present work, the electrocaloric properties of ferroelectric nanotubes are investigated using a phase field model as a function of strain, temperature, and electric field. We demonstrate that the large ECE induced by domain transition can be realized near room temperature in the ferroelectric nanotubes epitaxially grown on a substrate as shown in Fig. 1, in which the misfit strain plays a critical role.

\section{Results}

Temperature-dependent domain transition and phase transition. Fig. 2 shows the $3 \mathrm{D}$ polarization distributions with different temperatures and electric fields in the ferroelectric nanotubes subjected to an in-plane compressive strain of $\varepsilon_{11}=\varepsilon_{22}=$ $-0.39 \%$. Due to the existence of top and bottom dead layers, the ferroelectric nanotube is close to an open-circuit boundary condition. In the absence of electric field, polarizations form a vortex structure for all given temperatures, in which the vortex axis coincides with the $x_{3}$ axis as shown in Fig. 2 (a) and (b). The vortex structure reduces depolarization energy greatly through the head-to-tail arrangement of polarization vectors in the $x_{1}-x_{2}$ plane. However, the polarizations form a multi-domain pattern with $90^{\circ}$ domain wall in the appearance of electric field when the temperature is lower than $30^{\circ} \mathrm{C}$, as shown in Fig. 2 (c). In the multi-domain state, most polarizations are along the direction of electric field to reduce the external electric energy. Nevertheless, some polarizations are in the $x_{1}-x_{2}$ plane to decrease the elastic energy through the formation of $90^{\circ}$ domain wall. When the temperature increases, all polarizations are along the direction of electric field and form a single domain, which is induced by the decrease of polarization magnitudes. For small polarization magnitude, the contribution of elastic energy to the whole system becomes weaker. The single domain state with smaller polarization magnitude will not induce large elastic energy. The transition from multiply domain state to single domain is a consequence of the minimization of total free energy in the system, which involves a dedicated interplay among the electric field, strain, temperature and polarization. During the adiabatic depolarization process, the polarization decreases in the direction of electric field and domain transition takes place. In the absence of the electric field, the domain transition doesn't lead to heating during the spontaneous depolarization process.

In order to investigate the effect of strain on the domain transition, Fig. 3 gives the polarization distributions with different temperatures and electric fields in the ferroelectric nanotubes subjected to an

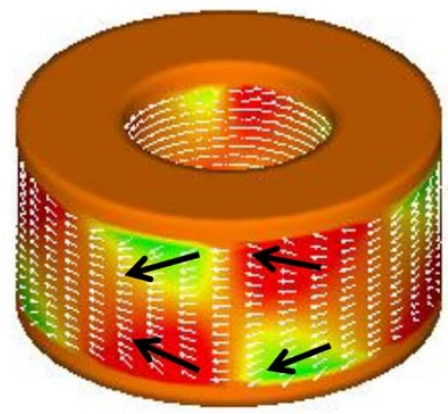

(a) $\mathrm{E}_{3}{ }^{*}=0, \mathrm{~T}=25^{\circ} \mathrm{C}$

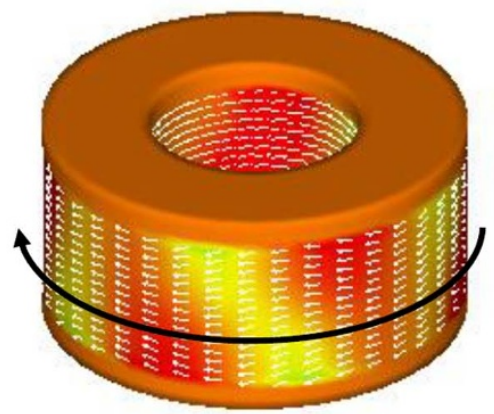

(b) $\mathrm{E}_{3}{ }^{*}=0, \mathrm{~T}=30^{\circ} \mathrm{C}$

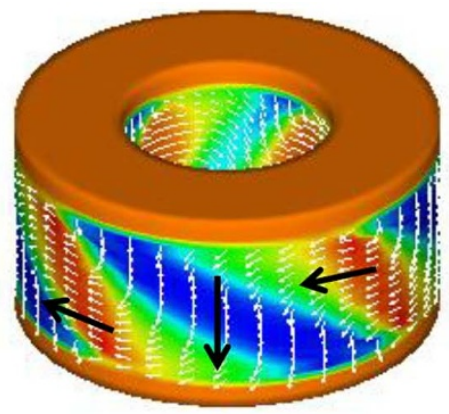

(c) $\mathrm{E}_{3}{ }^{*}=0.5, \mathrm{~T}=25^{\circ} \mathrm{C}$

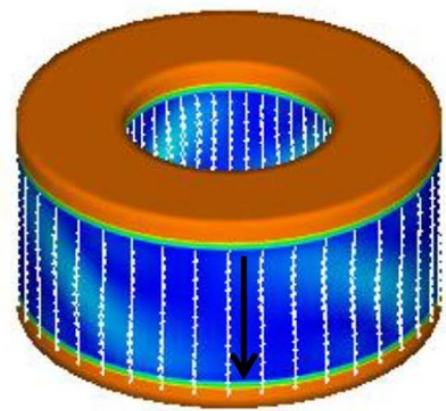

(d) $\mathrm{E}_{3}{ }^{*}=0.5, \mathrm{~T}=30^{\circ} \mathrm{C}$
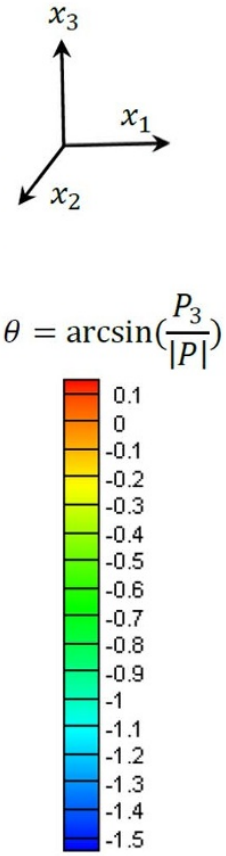

0.1

$-0.2$

0.4
-0.5

0.6

$-8$

$-0.9$

$-1.1$

$-1.3$

$-1.5$

Figure 2 The polarization distributions of ferroelectric nanotube under different electric fields and temperatures when the in-plane compressive strain is $\boldsymbol{\varepsilon}_{\mathbf{1 1}}=\boldsymbol{\varepsilon}_{\mathbf{2}}=\mathbf{- 0 . 3 9 \%}$. (a) and (b) show the polarization distributions without external electric field at $25^{\circ} \mathrm{C}$ and $30^{\circ} \mathrm{C}$, respectively; (c)

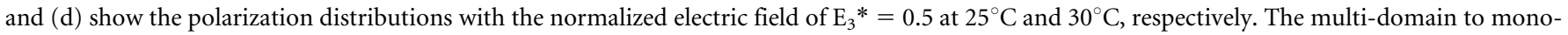

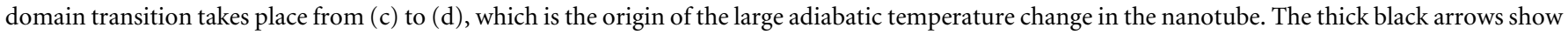
the direction of polarizations in a domain and the small white arrows denote local polarization vectors. 


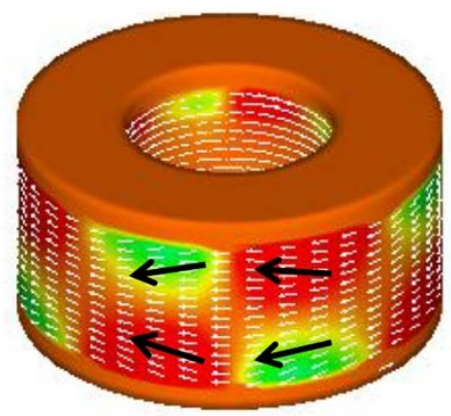

(a) $\mathrm{E}_{3}{ }^{*}=0, \mathrm{~T}=25^{\circ} \mathrm{C}$

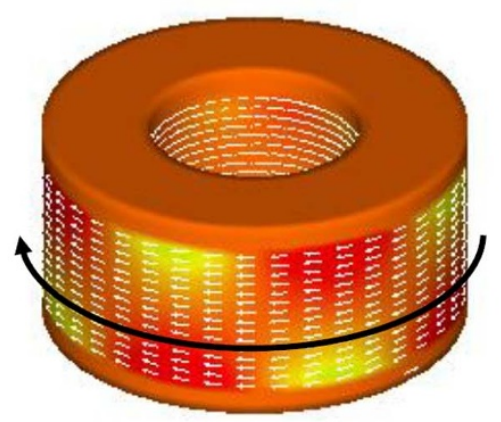

(b) $\mathrm{E}_{3}{ }^{*}=0, \mathrm{~T}=30^{\circ} \mathrm{C}$

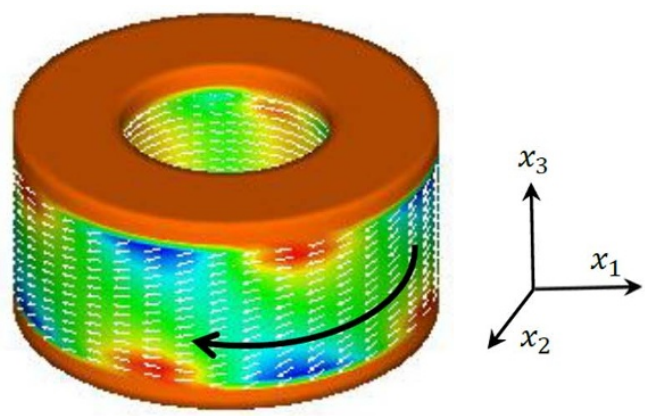

(c) $\mathrm{E}_{3}{ }^{*}=0.5, \mathrm{~T}=25^{\circ} \mathrm{C}$

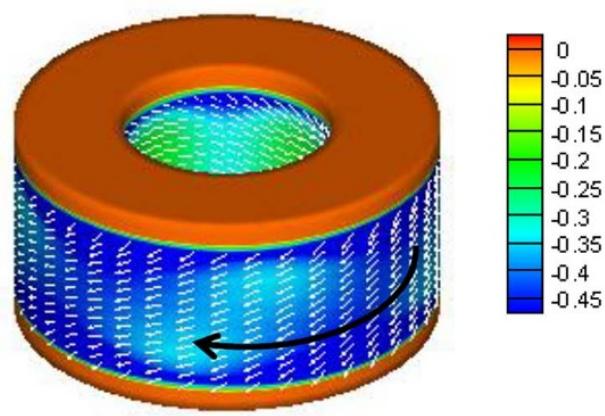

(d) $\mathrm{E}_{3}{ }^{*}=0.5, \mathrm{~T}=30^{\circ} \mathrm{C}$

Figure 3 The polarization distributions of ferroelectric nanotube under different electric fields and temperatures when the in-plane tensile strain is $\varepsilon_{11}=\varepsilon_{22}=0.39 \%$. (a) and (b) show the polarization distributions without external electric field at $25^{\circ} \mathrm{C}$ and $30^{\circ} \mathrm{C}$, respectively; (c) and (d) show the polarization distributions with the external electric field of $\mathrm{E}_{3}{ }^{*}=0.5$ at $25^{\circ} \mathrm{C}$ and $30^{\circ} \mathrm{C}$, respectively. The thick black arrows show the direction of polarizations in the polarization vortex.

in-plane tensile strain of $\varepsilon_{11}=\varepsilon_{22}=0.39 \%$. When the electric field is absent, polarizations form vortex structures for all given temperatures, which are similar to those of compressive strain in Fig. 2. When an electric field is applied, there is no $90^{\circ}$ domain structure due to the effect of tensile strain in the $x_{1}-x_{2}$ plane. However, there exists polarization component in $x_{3}$ direction induced by electric field for all given temperatures although the polarizations do not form a multidomain state like Fig. 2(c). The absence of multi-domain state under a tensile strain indicate that the strain play an essential role in the domain transition.

To understand the temperature dependence of polarization, Fig. 4 shows the changes in polarization magnitude $|P|$ and polarization component $\mathrm{P}_{3}$ in the nanotubes when temperature increases, which are the average values for all nodes. When temperature is less than $25^{\circ} \mathrm{C}$, polarization magnitudes $|P|$ and polarization component $\mathrm{P}_{3}$ have almost the same value as indicated in Fig. 4 (a) (b) (c).When temperature increases to $30^{\circ} \mathrm{C}$, the polarization magnitudes decrease shapely and then become stable for all cases. Nevertheless, the stable values of polarization magnitude are different for all cases. For the case of compressive in-plane strain, the stable values of polarization magnitude have a large difference under different electric fields. In the absence of electric field, the value is close to zero, which indicates the system becomes paraelectric phase. The appearance of phase transition from ferroelectric to paraelectric state at $30^{\circ} \mathrm{C}$ is attributed to the size effect of the nanotube. When the electric field increases, polarization magnitudes would increase. On the other hand, the values of polarization magnitude are close for all different electric fields, when the in-plane strain becomes tensile. Interestingly, the polarization magnitude is close to $0.2 \mathrm{C} / \mathrm{m}^{2}$ even the temperature is higher than Curie temperature in the absence of electric field, which implies the in-plane tensile strain can induce the in-plane polarizations. When the strains are absent, the trend of polarization change with temperatures is similar to that of tensile strain. Fig. 4 (d) (e) and (f) give temperature dependences of polarization component $\mathrm{P}_{3}$ for those cases. $\mathrm{P}_{3}$ decreases suddenly when temperature increases to $30^{\circ} \mathrm{C}$ for the compressive strain. The abrupt change is induced by the phase transition and domain transition at the same time, which will influence the adiabatic temperature change. When the temperature increases to $300^{\circ} \mathrm{C}, \mathrm{P}_{3}$ has less change for all cases.

Adiabatic temperature change near room temperature. In order to understand the change of the derivative of polarization with respect to temperature, Fig. 5 (a) (b) and (c) give the variation of the average value of $\mathrm{d} P_{3} / \mathrm{dT}$ under different electric fields in the ferroelectric nanotubes subjected to the compressive strain of $\varepsilon_{11}=\varepsilon_{22}=$ $-0.39 \%$, the tensile strain of $\varepsilon_{11}=\varepsilon_{22}=0.39 \%$, and without strain, respectively. It is found that the maximum value of $\mathrm{dP}_{3} / \mathrm{dT}$ appears near room temperature, which is close to the corresponding domain transition temperature and ferroelectric transition temperature for both the compressive and tensile strains. However, the variation of $\mathrm{dP}_{3} / \mathrm{dT}$ is quite small when the temperature is larger than room temperature, as shown in Fig. 5. Interestingly, for the compressive strain of Fig. 5 (a), the average value of $\mathrm{dP}_{3} / \mathrm{dT}$ is negative in the whole temperature range while it is positive for the case of tensile strain. Furthermore, its magnitude is much larger than that of Fig. 5 (b). The positive $\mathrm{dP}_{3} / \mathrm{dT}$ under tensile strain may be due to the fact that the polarization vectors are easier to rotate orientations along the applied electric with a smaller magnitude at high temperature. Due to the influence of $\mathrm{dP}_{3} / \mathrm{dT}$, the maximum values of the adiabatic temperature changes are $10.3 \mathrm{~K}$ and $-0.5 \mathrm{~K}$ for the compressive strain of $\varepsilon_{11}=\varepsilon_{22}=-0.39 \%$ and the tensile strain of $\varepsilon_{11}=\varepsilon_{22}=$ $0.39 \%$, respectively, which appear around the phase transition temperature and domain transition temperature, as shown in Fig. 6(a) and (b). The adiabatic temperature change induced by domain transition in ferroelectric nanotubes under a compressive strain is 3 times larger than that of ferroelectric thin film ${ }^{19}$. When 
(a)
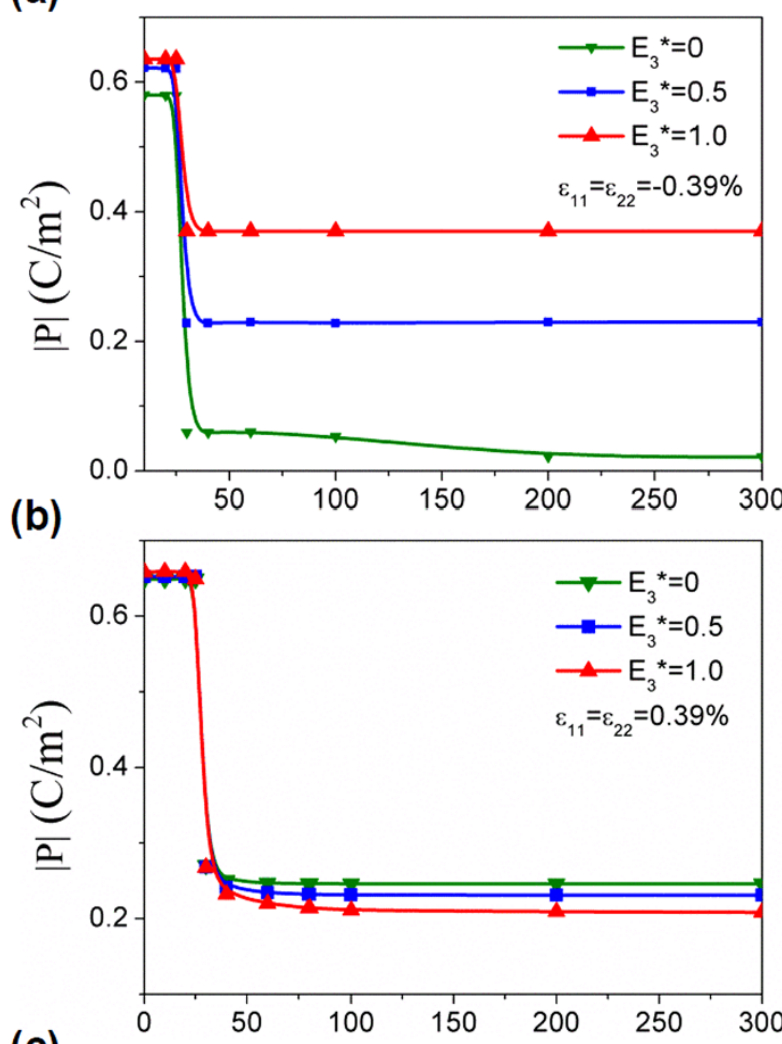

(c)

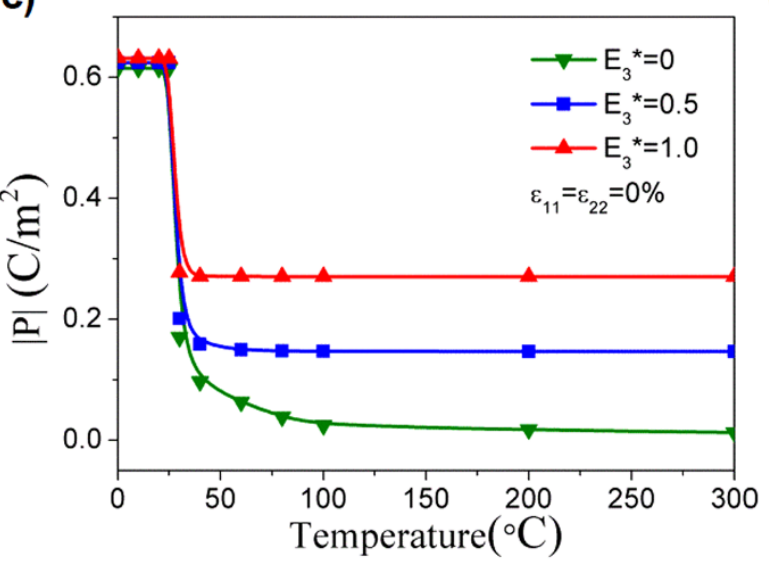

(d)

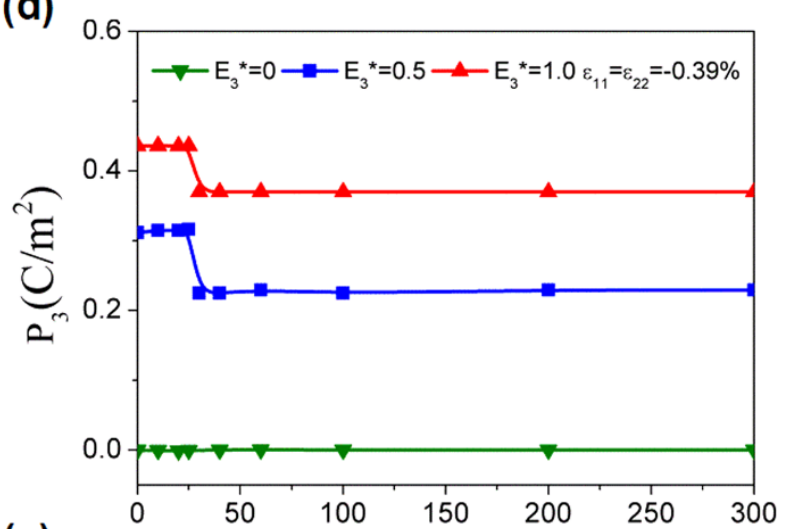

(e)

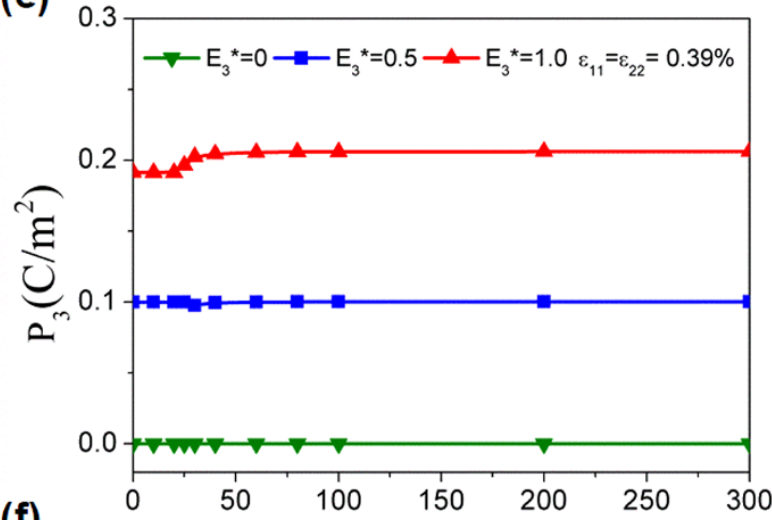

(f)

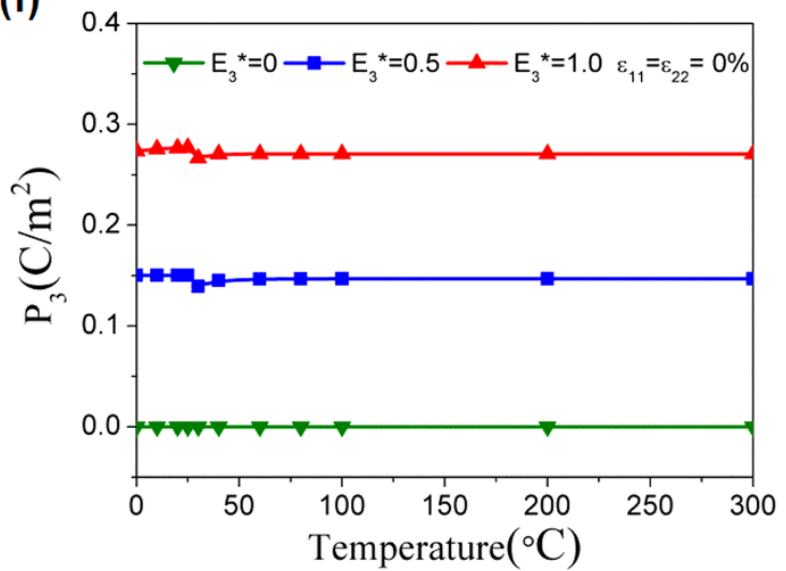

Figure $4 \mid$ Temperature dependence of the averaged polarization magnitude $|P|$ and polarization component $\mathrm{P}_{3}$ along with the direction of the electric field $\mathrm{E}_{3}$ in the ferroelectric nanotube subjected to different electric fields. (a) and (d) with the in-plane compressive strain of $\varepsilon_{11}=\varepsilon_{22}=-0.39 \%$; (b) and (e) with the in-plane tensile strain of $\varepsilon_{11}=\varepsilon_{22}=0.39 \%$; (c) and (f) without strain.

the strain is absent, the largest adiabatic temperature change is $1.4 \mathrm{~K}$ as shown in Fig. 6 (c), which is induced by the ferroelectric transition. These results imply that the domain transition is responsible for the giant ECE of ferroelectric nanotube that appears only under the compressive strains, which is consists with the influence of domain transition on the electrocaloric properties of $2 \mathrm{D} \mathrm{PbTiO}_{3}$ model ${ }^{21}$. Moreover, the maximum value of the temperature change increases with the increase of the electric field for both cases.

Influence of strains on the adiabatic temperature change. To further investigate the influence of strain on the adiabatic temperature change, Fig. 7 shows the temperature dependence of adiabatic temperature changes under different electric fields in the ferroelectric nanotubes with different tensile and compressive strains, respectively. For all curves of Fig. 7, there is a peak which is near room temperature. When the in-plane compressive strain is applied, the maximum adiabatic temperature change of the nanotubes increases, as shown in
Fig. 7 (a) and (b). The maximum adiabatic temperature change reach 4.1 $\mathrm{K}$ and $7.7 \mathrm{~K}$ due to the domain transition for the compressive strains of $\varepsilon_{11}=\varepsilon_{22}=-0.124 \%$ and $\varepsilon_{11}=\varepsilon_{22}=-0.248 \%$, respectively, which are several times larger than that without strain. Because there is no domain transition in the nanotube under a tensile strain, the maximum adiabatic temperature changes are smaller than that without strain, as indicated by Fig. 7 (c)-(d) and Fig. 6(c). Furthermore, the maximum adiabatic change decreases when the tensile strain increases from $\varepsilon_{11}=\varepsilon_{22}=0.124 \%$ and $\varepsilon_{11}=\varepsilon_{22}=$ $0.248 \%$. The strain dependence of adiabatic temperature change suggests that the ECE of ferroelectric nanotubes can be effectively tuned by the external strain.

\section{Discussion}

In in this work, we have shown that a giant adiabatic temperature change near room temperature in the ferroelectric nanotubes 
(a)

(b)

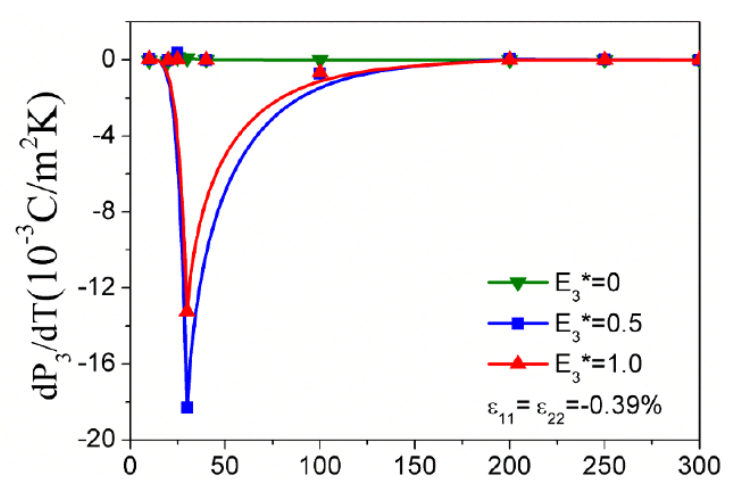

(c)
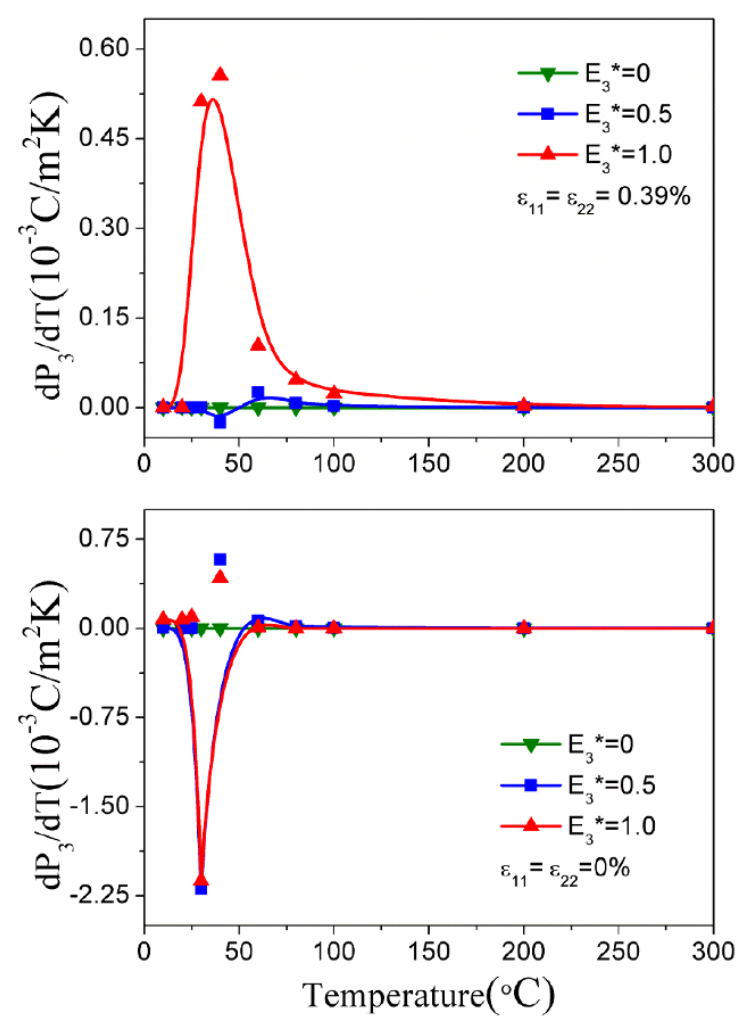

Figure $5 \mid$ The temperature dependences of $\mathrm{dP}_{3} / \mathrm{dT}$ for the ferroelectric nanotubes subjected to (a) the in-plane compressive strain of $\varepsilon_{11}=\varepsilon_{22}=$ $-0.39 \%$. (b) the in-plane tensile strain of $\varepsilon_{11}=\varepsilon_{22}=0.39 \%$;

(c)without strain. The maximum values of $\mathrm{dP}_{3} / \mathrm{dT}$ appear at the temperature $25^{\circ} \mathrm{C}$, which is close to the corresponding domain transition and phase transition temperatures.

subjected to an in-plane compressive strain. The giant adiabatic temperature is induced by the extrinsic contribution from multi-domain to mono-domain transition, which is much larger than the intrinsic one. The domain transition from a multiply domain state to single domain takes place with the minimization of total free energy, which involves a dedicated interplay among the electric field, strain, temperature and polarization. The epitaxial strain induced by the mismatch between the nanotube and substrate plays an essential role in the domain transition, which can be used to tune the magnitude of the adiabatic temperature change. Careful selection of external strain allows us to harness the extrinsic contribution of domain transition to obtain large adiabatic temperature change near room temperature. The present work may stimulate experimental investigations of perovskite ferroelectric nanotubes as solid state cooling components, in addition to theoretical studies of the interplay of couplings among the electric field, strain, temperature and polarization degrees of freedom. (a)

(b)

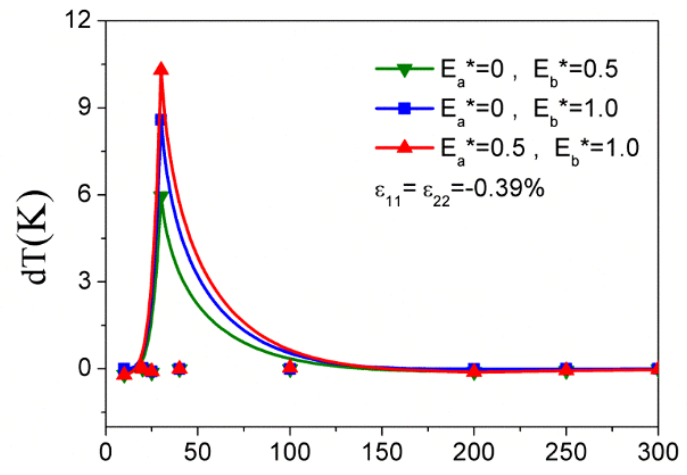

(c)
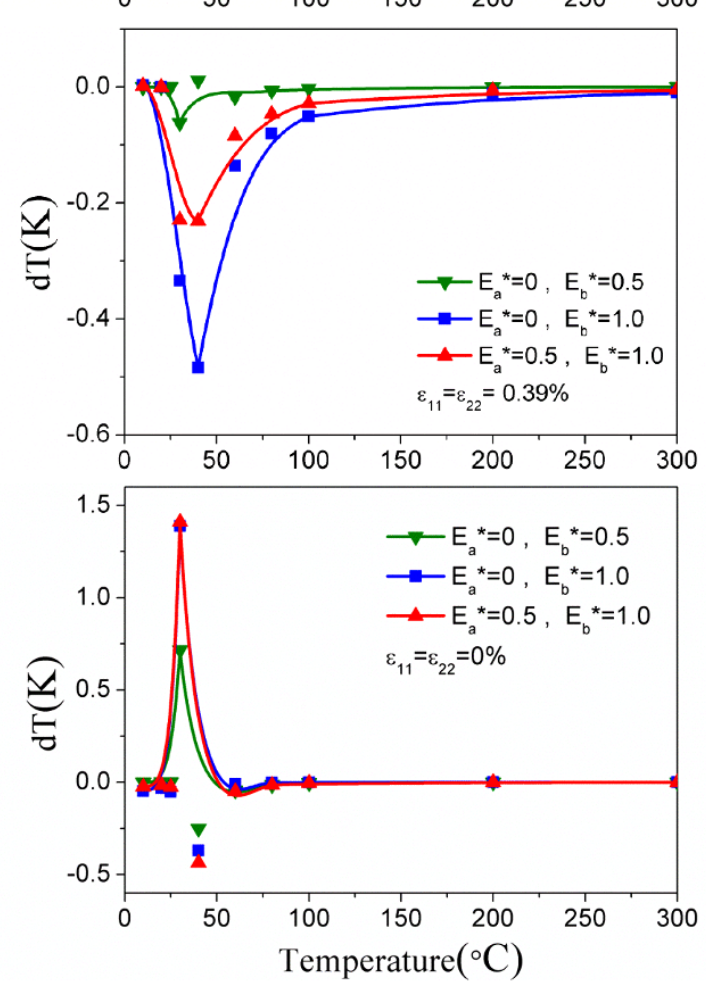

Figure 6 | Temperature dependence of the averaged adiabatic temperature change under different electric fields of $\mathrm{E}^{*}=0.5$ and $\mathrm{E}^{*}=$ 1.0 in the ferroelectric nanotube subjected to (a) the in-plane compressive strain of $\varepsilon_{11}=\varepsilon_{22}=-0.39 \%$; (b) the in-plane tensile strain of $\varepsilon_{11}=\varepsilon_{22}=$ $0.39 \%$; (c) without strain.

\section{Methods}

Simulation methodology. To model the ferroelectric phase transition and domain transition, polarization $\boldsymbol{P}=\left(P_{1}, P_{2}, P_{3}\right)$ is used as the order parameter based on the Landau-Devonshire theory. Differing from the homogeneous assumption in monodomain thermodynamic model, the thermodynamic free energy in the phase-field model is described in terms of spatially continuous but inhomogeneous polarization, in which the nonlocal energy of polarization gradient is explicitly taken into account. The phase transition and domain transition are direct consequences of the minimization process of the total free energy of the entire system. The total free energy of the ferroelectric is usually obtained by integrating an electrical enthalpy over the whole volume, which can be expressed $\mathrm{as}^{23}$

$$
\begin{aligned}
f\left(P_{i}, P_{i, j}, \varepsilon_{i j}, E_{i}\right) & =\alpha_{i} P_{i}^{2}+\alpha_{i j} P_{i}^{2} P_{j}^{2}+\alpha_{i j k} P_{i}^{2} P_{j}^{2} P_{k}^{2}+\frac{1}{2} c_{i j k l} \varepsilon_{i j} \varepsilon_{k l}-q_{i j k l} \varepsilon_{i j} P_{k} P_{l} \\
& +\frac{1}{2} g_{i j k l} P_{i, j} P_{k, l}-\frac{1}{2} \varepsilon_{0} E_{i} E_{i}-E_{i} P_{i}
\end{aligned}
$$

In Eq. (1), the first three terms represent the Landau energy, where $\alpha_{i}$ is the dielectric stiffness, $\alpha_{i j}$ and $\alpha_{i j k}$ are higher order dielectric stiffnesses. The fourth term denotes the elastic energy of the system, where $\varepsilon_{i j}$ and $c_{i j k l}$ are the strains and elastic constants, respectively. The fifth term denotes the coupling energy between the polarizations and strains, where $q_{i j k l}$ are the electrostrictive coefficients. The sixth term is the gradient energy, i.e. the domain wall energy, where $g_{i j k l}$ are the gradient 
(a)

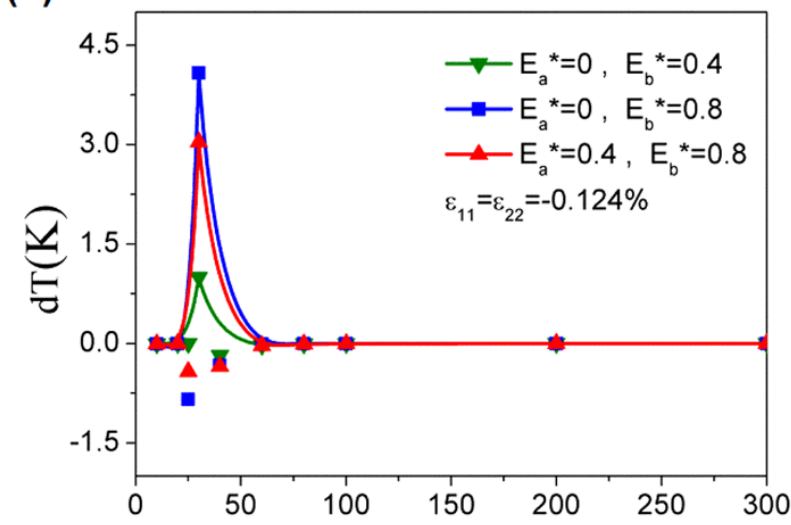

(b)

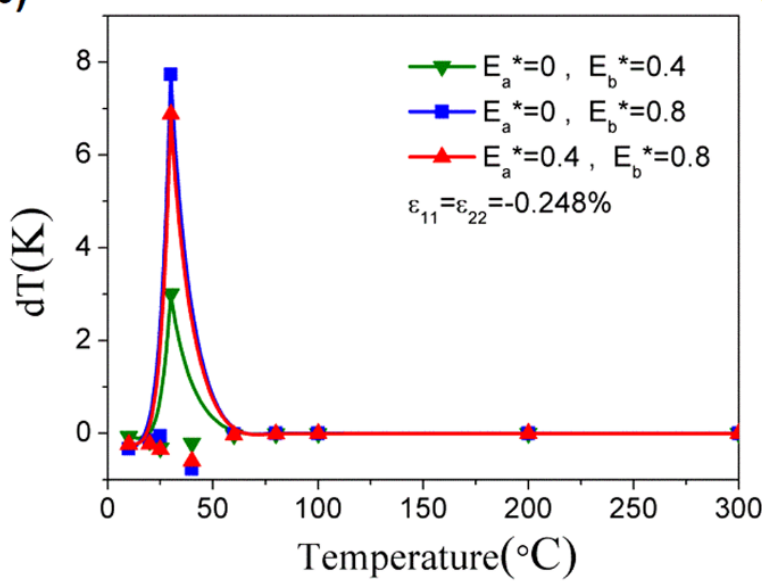

(c)

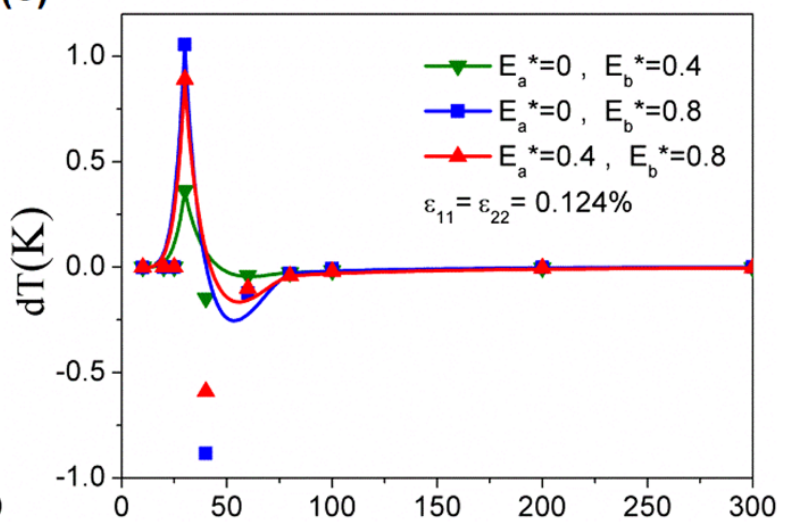

(d)

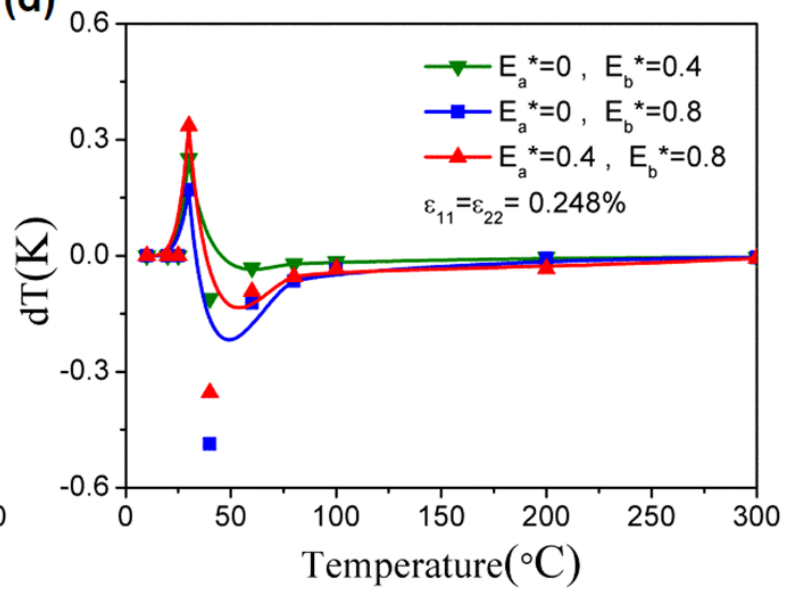

Figure $7 \mid$ Temperature dependence of averaged temperature change under different electric fields of $\mathrm{E}_{3}{ }^{*}=0.4$ and $\mathrm{E}_{3} *=0.8$ in the ferroelectric nanotube subjected to (a) and (b) the compressive strains; (c) and (d) the tensile strains.

coefficients and $P_{i, j}$ denotes the spatial derivative of the $i$ th component of the polarization vector, $P_{i}$, with respect to the $j$ th coordinate and $i, j=1,2,3$. The gradient energy gives the energy penalty for spatially inhomogeneous polarization. The last terms are the electric energy density due to the presence of electric field, in which $\varepsilon_{0}$ is the dielectric constant of the vacuum and $E_{i}$ is the total electric field including the external electric field and depolarization field. With the electrical enthalpy of of Eq. (1), the stresses and electric displacements can be derived as $\sigma_{i j}=\partial f / \partial \varepsilon_{i j}$ and $D_{i}=$ $-\partial f / \partial E_{i}$ respectively.

In the phase field model, the temporal evolution of spatially inhomogeneous polarization can be obtained from the following time-dependent Ginzburg-Landau equation ${ }^{23}$

$$
\frac{\partial P_{i}(r, t)}{\partial t}=-L \frac{\delta F}{\delta P_{i}(r, t)} \quad(i=1,2,3),
$$

where $L$ is the kinetic coefficient, $F=\int_{V} f d v$ is the total free energy of the simulated system. The variation of $\frac{\delta F}{\delta P_{i}(r, t)}$ at the right hand side of Eq. (2) represents the thermodynamic driving force for the spatial and temporal evolution of polarization, $\boldsymbol{r}=\left(x_{1}, x_{2}, x_{3}\right)$ denotes the spatial vector and $t$ is time. In addition to Eq. (2), the mechanical and electrical equilibrium equations of $\sigma_{i j, j}=0$ and $D_{i, i}=0$ must be satisfied at the same time for charge and body force free ferroelectric materials. To solve Eq. (2) by the finite element method, an eight-node brick element with seven degrees of freedom at each node is employed for the space discretization, and the backward Euler iteration method is adopted for the time integration ${ }^{23-25}$. In the presence of an external electric field, the adiabatic temperature change is calculated by $^{18}$

$$
\Delta T=-T \int_{E_{a}}^{E_{b}} \frac{1}{C_{E}(T, E)}\left(\frac{\partial P_{i}}{\partial T}\right)_{E} d E_{i}
$$

where $C_{E}(T, E)$ is the heat capacity per unit volume at constant electric field. Following Karthik and Martin ${ }^{19}$, the contribution of the polarization to the heat capacity is neglected in the present work. The absolute value of the heat capacity of $\mathrm{PbTiO}_{3}$ is approximately constant as $3.0 \times 10^{6} \mathrm{~J} / \mathrm{m}^{3} \mathrm{~K}$ in our calculation.

Simulation model. To demonstrate the large ECE near room temperature induced by domain transition, $\mathrm{PbTiO}_{3}$ ferroelectric nanotube epitaxially grown on a substrate is investigated as shown in Fig. 1. The substrate can provide tensile or compressive inplane strain for the ferroelectric nanotubes through lattice or thermal mismatch. The top and bottom of the nanotube are covered by metal electrodes, on which different electrical potentials are given to apply different electric fields. In order to consider the material degradation at electrode-ferroelectrics interface, two dielectric layers at the upper and lower interfaces, i.e. the common "deadlayers" in ferroelectric capacitors, are included in the model tube. The inner and outer radii of the nanotube are $8 \mathrm{~nm}$ and $16 \mathrm{~nm}$, respectively. The height of the nanotube is $16 \mathrm{~nm}$ including the deadlayers. In the simulation, we employ 8000 discrete brick elements to model the nanotube. All the materials constants used in the simulation are the same as those of Ref. 21. For convenience, all the materials parameters are normalized and the normalized equations are solved. The normalized formula in the present study is the same as that used in the previous work ${ }^{21}$. The superscript * represents the normalized value in this paper. The stable domain structures are simulated in the ferroelectric nanotubes with different misfit strains, temperatures and electric fields. The in-plane compressive and tensile strains are applied to the nanotube by giving tensile and compressive displacements, respectively, to all nodes in the top surface and fixing the vertical displacement of all nodes at the bottom interface. To obtain the stable polarization distribution at each temperature, the time step is set as $\Delta t^{*}=0.04$ and the total numbers of steps is taken as 2000 .

1. Kobeko, P. P. \& Kurchatov, I. V. Dielectric properties of ferroelectrics. Z. Phys. 66, 192 (1930).

2. Childress, J. D. Application of a Ferroelectric Material in an Energy Conversion Device. J. Appl. Phys. 33, 1793-1798 (1962).

3. Fatuzzo, E., Kiess, H. \& Nitsche, R. Theoretical Efficiency of Pyroelectric Power Converters. J. Appl. Phys. 37, 510-516 (1966).

4. Thacher, P. D. Electrocaloric Effects in Some Ferroelectric and Antiferroelectric $\mathrm{Pb}(\mathrm{Zr}, \mathrm{Ti}) \mathrm{O}_{3}$ Compounds. J. Appl. Phys. 39, 1996-2002 (1968).

5. Mischenko, A. S., Zhang, Q., Scott, J. F., Whatmore, R. W. \& Mathur, N. D. Giant Electrocaloric Effect in Thin-Film $\mathrm{PbZr}_{0.95} \mathrm{Ti}_{0.05} \mathrm{O}_{3}$. Science 311, 1270-1271 (2006).

6. Morozovska, A. N., Glinchuk, M. D. \& Eliseev, E. A. Phase transitions induced by confinement of ferroic nanoparticles. Phys. Rev. B. 76, 014102 (2007).

7. Spanier, J. E. et al. Ferroelectric Phase Transition in Individual Single-Crystalline $\mathrm{BaTiO}_{3}$ Nanowires. Nano. Lett. 6, 735-739 (2006). 
8. Hong, J. W. \& Fang, D. N. Size-dependent ferroelectric behaviors of $\mathrm{BaTiO}_{3}$ nanowires. Appl. Phys. Lett. 92, 012906 (2008).

9. Scott, J. F. Cylinder stress in nanostructures: effect on domains in nanowires, nanotubes, and nano-disks. J. Phys.: Condens. Matter 26, 212202 (2014).

10. Hong, J. W., Catalan, G., Fang, D. N., Artacho, E. \& Scott, J. F. Topology of the polarization field in ferroelectric nanowires from first principles. Phys. Rev. B. 81, 172101 (2010).

11. Akcay, G., Alpay, S. P., Mantese, J. V. \& Rossetti, G. A. Magnitude of the intrinsic electrocaloric effect in ferroelectric perovskite thin films at high electric fields. Appl. Phys. Lett. 90, 012906 (2008).

12. Akcay, G., Alpay, S. P., Rossetti, G. A. \& Scott, J. F. Influence of mechanical boundary conditions on the electrocaloric properties of ferroelectric thin films J. Appl. Phys. 103, 024104 (2008).

13. Li, B., Wang, J. B., Zhong, X. L., Wang, F. \& Zhou, Y. C. Room temperature electrocaloric effect on $\mathrm{PbZr}_{0.8} \mathrm{Ti}_{0.2} \mathrm{O}_{3}$ thin film. J. Appl. Phys. 107, 014109 (2010).

14. Liu, Y., Infante, I. C., Lou, X. J., Lupascu, D. C. \& Dkhil, B. Giant mechanicallymediated electrocaloric effect in ultrathin ferroelectric capacitors at room temperature. J. Appl. Phys. 104, 012907 (2014).

15. Morrison, F. D. et al. Ferroelectric Nanotubes. Rev. Adv. Mater. Sci. 4, 114-122 (2003).

16. Morrison, F. D., Ramsay, L. \& Scott, J. F. High aspect ratio piezoelectric strontiumbismuth-tantalate nanotubes. J. Phys.: Cond. Matter 15, 527-532 (2003).

17. Hernandez-Sanchez, B. A. et al. Examination of size-induced ferroelectric phase transitions in template synthesized $\mathrm{PbTiO}_{3}$ nanotubes and nanofibers. Chem. Mater. 17, 5909-5919 (2005)

18. Scott, J. F. Electrocaloric Materials. Rev. Mater. Sci. 41, 229-240 (2011).

19. Karthik, J. \& Martin, L. W. Effect of domain walls on the electrocaloric properties of $\mathrm{Pb}\left(\mathrm{Zr}_{1-\mathrm{x}}, \mathrm{Ti}_{\mathrm{x}}\right) \mathrm{O}_{3}$ thin films. Appl. Phys. Lett. 99, 032904 (2011).

20. Bai, Y., Han, X., Ding, K. \& Qiao, L. J. Combined effects of diffuse phase transition and microstructure on the electrocaloric effect in $\mathrm{Ba}_{1-}{ }_{\mathrm{x}} \mathrm{Sr}_{\mathrm{x}} \mathrm{TiO}_{3}$ ceramics. Appl. Phys. Lett. 103, 162902 (2013).

21. Wang, J. et al. Large electrocaloric effect induced by the multi-domain to monodomain transition in ferroelectrics. J. Appl. Phys. 115, 164102 (2014).

22. Dai, X., Cao, H. X., Jiang, Q. \& Lo, V. C.H. Influence of thermal strains on the electrocaloric and dielectric properties of ferroelectric nanoshells. J. Appl. Phys 106, 034103 (2009).
23. Wang, J. \& Kamlah, M. Domain control in ferroelectric nanodots through surface charges. Appl. Phys. Lett. 93, 042906 (2008).

24. Wang, J. \& Zhang, T. Y. Phase Field Study of Polarization Vortex in Ferroelectric Nanostructures. Journal of Advanced Dielectrics 02, 1241002 (2012).

25. Wang, J., Shu, W., Shimada, T., Kitamura, T. \& Zhang, T. Y. Role of grain orientation distribution in the ferroelectric and ferroelastic domain switching of ferroelectric polycrystals. Acta. Mater. 61, 6037-6049 (2013).

\section{Acknowledgments}

The authors acknowledge the financial support from the National Natural Science Foundation of China (Grant No. 11321202, 11472242), the Fundamental Research Funds for the Central Universities (2014FZA4027).

\section{Author contributions}

J.W. conceived and designed the concept. M.L. conducted the simulations. M.L. and J.W. wrote the paper.

\section{Additional information}

Competing financial interests: The authors declare no competing financial interests.

How to cite this article: Liu, M. \& Wang, J. Giant electrocaloric effect in ferroelectric nanotubes near room temperature. Sci. Rep. 5, 7728; DOI:10.1038/srep07728 (2015).

This work is licensed under a Creative Commons Attribution-NonCommercialNoDerivs 4.0 International License. The images or other third party material in this article are included in the article's Creative Commons license, unless indicated otherwise in the credit line; if the material is not included under the Creative Commons license, users will need to obtain permission from the license holder in order to reproduce the material. To view a copy of this license, visit http:// creativecommons.org/licenses/by-nc-nd/4.0/ 